\title{
Politique
}

\section{Un syndicalisme non-partisan durant les années péquistes}

\section{Roch Denis et Serge Denis}

Numéro 10, automne 1986

Opinions et votes

URI : https://id.erudit.org/iderudit/040535ar

DOI : https://doi.org/10.7202/040535ar

Aller au sommaire du numéro

Éditeur(s)

Société québécoise de science politique

ISSN

0711-608X (imprimé)

1918-6584 (numérique)

Découvrir la revue

Citer cette note

Denis, R. \& Denis, S. (1986). Un syndicalisme non-partisan durant les années péquistes. Politique, (10), 91-116. https://doi.org/10.7202/040535ar d'utilisation que vous pouvez consulter en ligne.

https://apropos.erudit.org/fr/usagers/politique-dutilisation/ 


\title{
UN SYNDICALISME NON-PARTISAN DURANT LES ANNÉES PÉQUISTES
}

\author{
Roch Denis, Université du Québecà Montréal \\ Serge Denis, Université d'Ottawa
}

\section{Introduction}

Il y a un an, le 2 décembre 1985, le Parti québécois subissait la défaite aux élections générales. Fin d'une période, début d'une autre? On s'entend généralement pour reconnaître que ce résultat a signifié davantage que le simple remplacement d'un parti par un autre au pouvoir. Après le référendum de 1980, et le rapatriement de 1982 , le revers péquiste a pu symboliser le dernier acte d'une pièce moderne... commencée il y a une trentaine d'années. Les uns ont applaudi à tout rompre. D'autres, plus nombreux, ont quitté la salle déçus ou furieux.

Pour les syndicats qui sont les organisations sociales les plus nombreuses, la défaite péquiste entraîne inévitablement des conséquences importantes sur le plan politique.

Le texte qui suit veut tenter de les mesurer et de les apprécier, en reconnaissant que l'action, l'orientation et les prises de position de mouvement ouvrier ont un impact sur la scène politique, quelque soient les formes qu'elles empruntent.

Mais avant d'étudier les lendemains d'une défaite, nous pensons qu'il faut revenir sur le type d'alliance qui s'est nouée historiquement entre le PQ et les syndicats au Québec. 
Notre but à cet égard n'est ni de faire une contribution à l'analyse théorique des rapports entre syndicats et partis, ni de faire un bilan du lien spécifique qui s'est établi entre nationalisme, péquisme et développement politique du syndicalisme.

De façon beaucoup plus limitée, nous nous interrogeons sur l'évolution qu'a connue l'orientation politique de la non-partisanerie ${ }^{1}$ syndicale pendant les années péquistes. Plutôt qu'un texte fini, nous présentons des notes qui consignent un certain nombre de réflexions à partir de points de repères chronologiques.

Il ne s'agira pas ici de démontrer une évidence!... à savoir que les syndicats au Québec ont continué depuis deux décennies à pratiquer la non-partisanerie, à l'exemple du modèle nordaméricain prédominant. Nous tenterons plutôt de faire ressortir si et dans quelle mesure l'avènement du PQ a entraîné un renouvellement des pratiques politiques non-partisanes et le cas échéant d'en identifier les principales manifestations. Sur cette base, en conclusion, nous essaierons d'indiquer pourquoi et comment, à notre avis, la non-partisanerie traditionnelle est aujourd'hui remise en question dans le mouvement syndical québécois, y compris dans certaines de ses sphères dirigeantes.

La présente contribution s'inscrit dans une étude plus large que les deux auteurs ont engagée sur l'évolution politique du syndicalisme québécois dans l'après-guerre, étude qui comporte, notamment, une évaluation comparative des formes d'action politique au Québec et au Canada anglais.

1. Nous définissons la non-partisanerie comme cet ensemble de pratiques politiques syndicales qui peuvent allier la pression, la participation, la définition de plateformes ou de programmes législatifs adressés aux partis existant ou aux gouvernements, l'appui partisan à telle ou telle formation ou à tel ou tel candidat ou encore la mobilisation contre tel gouvernement, parti ou candidat mais qui excluent la formation, par le mouvement syndical comme tel, d'un parti qui serait directement son bras politique indépendant. Historiquement, c'est aux États-Unis que le syndicalisme a le plus développé cette forme d'action politique au $\mathrm{Xx}^{\mathrm{c}}$ siècle: rappelons la formule classique de la non-partisanerie qui persiste: "récompensons nos amis, punissons nos ennemis". 


\section{Le nouvel essor du nationalisme}

Étudier les formes de l'action politique syndicale au Québec au cours des vingt dernières années, c'est élucider d'abord le rapport du mouvement ouvrier au nationalisme et au Parti québécois. À partir de 1968, le parti de René Lévesque va réussir à se construire et à se présenter comme le nouvel instrument d'intervention populaire sur la scène politique. La fin des années 1960 ouvre en ce sens une période d'hégémonie politique du nationalisme péquiste au sein du syndicalisme, comme des mouvements sociaux en général. Les mécanismes de la relation qui s'établit alors entre les syndicats et le PQ marquent un développement significatif dans les modes d'intervention et d'existence politique des organisations ouvrières.

Le nationalisme québécois de la dernière période a beaucoup été étudié. Nous ne voulons dégager et rappeler dans ce texte que les traits principaux de son articulation aux mouvements ouvriers et populaires.

La dynamique sociale ouverte avec la fin des années 1940 s'est essentiellement matérialisée dans l'expansion et les transformations sociologiques et numériques que connaît le syndicalisme, principalement entre 1956 et le début des années 1970. Le creuset de cette évolution, joint à l'enthousiasme et à l'énergie que suscitent les premiers débats, espoirs et réformes de la révolution tranquille, favorise également la radicalisation politique de toute une série de secteurs sociaux plus ou moins liés à la petite bourgeoisie et au monde étudiant (artiste, Mouvement laïque de langue française, création de l'Union générale des étudiants du Québec, etc...). Cela, dans un cadre où le poids objectif de la question nationale tend à accentuer l'attrait des solutions alternatives, et où l'échec du NPD-PSQ (issu des efforts de la FTQ vers un $3^{\mathrm{e}}$ parti) au Québec apparaît définitif. Ce contexte explique qu'une fraction importante de ces secteurs tendra à se regrouper autour d'un projet 
nationaliste radical, se situant en prolongement et à gauche de la révolution tranquille. Le RIN, formé dès le mois de septembre 1960, apparaîtra quelques années plus tard, comme la manifestation principale de ce courant, sur une plate-forme d'indépendance nationale, de laïcisme et, progressivement, de rapprochement en direction des mobilisations ouvrières et populaires.

Le nationalisme est bientôt amené à se présenter comme seul pôle alternatif au Parti libéral et à l'Union nationale. Seule option indépendante des vieux partis, il tend à canaliser les volontés et les énergies de ruptures politiques, ce qui, en retour, lui donne un contenu nouveau: de mouvement traditionnel d'élite, il devient un phénomène de masse ${ }^{2}$. Corollairement, il contribue à définir la perception populaire des grandes questions politiques, il est l'enveloppe politique des revendications sociales et démocratiques.

\section{La crise du bipartisme}

Il faut souligner que c'est d'abord en imposant son hégémonie sur le mouvement d'aspirations nationales que le $P Q$ va réussir à se façonner comme parti de masse, à renforcer et ponctuer la marche vers une formation indépendante des libéraux et des unionistes. Il se situe en prolongement dés développements déjà enclenchés, tout en leur assurant des promesses de succès inconnues jusque là (René Lévesque a été l'un des ministres les plus populaires de Jean Lesage). On ne peut expliquer autrement le rythme extraordinaire de son développement: il obtient $23,1 \%$ des voix deux ans après sa formation (en régime bipartite), 30,2\% en 1973 et prend le pouvoir huit années après avoir été créé avec $41.7 \%$ des

2. C'est ce fait, à notre avis, qui explique d'abord que le nationalisme québécois moderne se situe en discontinuité avec les spéculations philosophico-religieuse et les thèmes ultramontains qui avaient jusque là survécu dans les divers courants nationalistes. 
votes $^{3}$. En cours de route, la vague a eu raison des unionistes et des créditistes.

Les nombreuses analyses sur l'origine sociale du vote péquiste sont révélatrices des couches de la population auxquelles il sert de canal d'expression politique. L'évolution de son membership est cependant tout aussi significative. Dès 1974-75, le parti a franchi le cap des 100,000 membres, et il en compte un peu plus de 130,000 au moment de la campagne de 1976. La composition de ce membership (qui recoupe grosso modo celle de son électorat et se fonde sur les forces vives, populaires et syndiquées, qui donnent le ton à la vie sociale depuis le début des années 1960) comme le type d'engagement qui lui est demandé tranchent sur ceux des vieux partis. La victoire contre Bourassa va renforcer ces processus. En 1979, par exemple, le PQ compte quelque 200,000 membres; ce nombre est passé à 238,000 en avril 1980, et à près de 300,000 en mars $1981^{4}$.

La victoire péquiste de 1976 a présenté aussi d'autres caractéristiques. D'abord, elle ne s'inscrit pas dans le cadre du bipartisme traditionnel, ni même d'une simple reformulation de ce cadre comme cela fut le cas, par exemple, aux USA avec le passage en bloc du CIO au Parti démocrate dans les années 1930. Il y avait eu alors cristallisation d'une composition sociale spécifique à l'électorat démocrate, mais les nouveaux syndicats n'en déployaient pas moins leurs formes d'expression politique par le canal de l'un des vieux partis.

Au Québec, dans les années 1970, les forces populaires ont porté au gouvernement un nouveau parti. Personne, parmi les

3. Dans un contexte où, pour diverses raison d'ordre historique, sociologique et social, environ $20 \%$ du vote global apparaît inaccessible au PQ (minorités anglosaxonne et allophones anglicisées).

4. Chiffres tirés du livre de Graham Fraser, Le Parti québécois, Montréal, Libre Expression, 1984, pp. 391-398. À ce moment, le PQ est le parti à affiliation directe, des pays occidentaux, qui semble regrouper proportionnellement le plus grand nombre de membres. 
responsables du Parti québécois ou des unions ouvrières, ne présente alors cette formation comme parti des travailleurs ${ }^{5}$, c'est-à-dire comme une consécration de l'autonomie du mouvement ouvrier sur la scène politique face aux partis traditionnels. Mais il est évident que le PQ parce qu'il s'est développé en rupture avec le Parti libéral et l'Union nationale, est perçu comme jouant ce rôle.

Ces faits donnent non seulement une nouvelle impulsion aux attentes envers le Pari québécois, ils renforcent toute la confiance à son égard. L'année 1976 confirme et accentue en ce sens le monopole de représentation politique qu'exerce dorénavant le Parti québécois sur le mouvement ouvrier.

À ce sujet, on explique parfois ${ }^{6}$, que l'élection du PQ en 1976 se serait produite à contre courant des développements sociaux antérieurs, c'est-à-dire en contradiction avec le cycle des grèves et des grandes mobilisations syndicales. De cela, on tend à conclure que le nouveau gouvernement eut en quelque sorte la partie belle face au syndicalisme, vu que le mouvement revendicatif était en position de reflux... D'un côté, il est vrai que la courbe des grèves est alors déclinante (ce qui procède notamment des difficultés économiques du milieu de la décennie 1970) et que le PQ va réussir en partie à décrisper les rapports sociaux. Mais en même temps, il faut souligner que le vote en faveur du PQ est lui-même le produit de l'élévation des niveaux de lutte populaire dans toute la période précédente, plutôt que de leur recul. Les plus grands combats économiques, les grèves générales et illégales, etc...,

5. Appellation la plus répandue au Québec dans les années 1970 lorsqu'on veut se référer à l'axe d'un $3^{\circ}$ parti de classe. C'est ainsi, par exemple, qu'en appelant plus ou moins explicitement au vote péquiste en 1976, les syndicats ont donné la consique d'élire «le parti qui est le plus près de nos intérêts», cependant que René Lévesque écrivait cette même année: "le parti des travailleurs est une utopie "pernicieuse"... la naissance d'un parti des travailleurs diviserait à coup sûr les forces vives du Québec» (Le jour, 12 février 1976). Il n'y avait donc pas confusion dans les termes, ni d'un côté ni de l'autre: le PQ ne se présentait pas, et n'était pas considéré comme un parti ouvrier.

6. Notamment, L. Favreau et P. L'Heureux, Le projet de société de la CSN, Montréal, CFP-Vie ouvrière, 1984, pp. 118-119. 
n'ont pas conduit à la satisfaction des demandes; les aspirations nationales ébranlent la structure de l'État fédéral. Il y a les lois de contrôle des salaires, que la grève pan-canadienne du mois d'octobre 1976 n'a pu faire rappeler, cependant que les nouvelles conditions économiques rendent, par elles-mêmes, plus aléatoires les acquis à l'intérieur de chaque entreprise. Dans ce cadre, le mouvement social tente de se manifester au niveau politique par l'intermédiaire du parti qui se présente comme la seule véritable alternative, le Parti québécois.

D'un autre côté, aucun des organismes représentatifs du patronat n'appui en 1976 - ni en 1981, d'ailleurs, - le Parti québécois. Sa victoire est ressentie comme une victoire du vote populaire et ouvrier, contre les partis et les hommes/femmes politiques que la bourgeoisie reconnaît explicitement sien-nes. En ce sens, plutôt que d'être vue comme concomitante d'un recul des luttes syndicales, la victoire du Parti québécois en 1976 doit être davantage assimilée, croyons-nous, à un phénomène politique de type front populaire, et à ce que représente l'élection d'un gouvernement de front populaire.

À l'instar des fronts populaires, il se présente comme une alternative politique permettant de rompre avec les vieilles formules de l'alternance gouvernementale habituelle. La victoire sur ces formules, c'est la victoire du v'ote des masses par le parti qu'elles saisissent comme leur parti.

D'un autre côté, on sait que l'une des caractéristiques premières des fronts populaires est de coaliser en des équations gouvernementales particulières des partis qui originent de l'évolution propre du mouvement ouvrier (PC, PS, travaillistes, etc...) et des partis dont la nature historique procède du mouvement d'autres forces sociales (par exemple, en France et en Espagne au cours des années 1930, de la bourgeoisie républicaine et laïque). Les gouvernements ainsi formés ne sont pas ouvriers, même si la force électorale première de ces coalitions est d'origine ouvrière et populaire. Si 
la création du Parti québécois n'a pas été le produit d'une action propre des syndicats, leur mouvement se reconnaît majoritairement dans ce parti, cherche aussi à le pénétrer de ses préoccupations, ce que le parti accepte, voire favorise. L'alliance politique est en quelque sorte informelle, mais elle est aussi forte que les coalitions formelles vues en d'autres pays.

L'échec du référendum va ouvrir les premières brèches dans le dispositif hégémonique du PQ. Mais dès 1981, l'opposition au rapatriement unilatéral de l'AANB contribuera à freiner temporairement les interrogations sur l'orientation politique et stratégique du PQ et à resouder autour de lui le mouvement nationaliste face à l'offensive d'Ottawa. Au scrutin de 1981, le Parti québécois obtient un peu plus de $49 \%$ des voix, et c'est à ce moment que le nombre de ses membres oscille autour de 300,000. L'unanimité du soutien populaire au PQ en milieu francophone est telle que certains observateurs se demandent alors si les libéraux y conservent encore quelque base électorale stable et significative ${ }^{7}$.

\section{PQ - NPD}

La place que s'est taillé le Parti québécois comme nouvelle formation correspondait à des développements sociaux et politiques qui avaient cours dans l'ensemble du pays. La fin de la décennie 1960 et la première moitié des années 1970, par exemple, donnent lieu à la prise du pouvoir par le NPD dans trois provinces de l'Ouest et à son élévation au rang d'Opposition officielle en Ontario, première région industrielle. Ces développements politiques sont le produit, au moins partiel, de la montée des luttes sociales qui ont été analysées par la Commission fédérale d'enquête Woods (1968). L'impact particulier que connaîtra le PQ vient de la

7. Voir, par exemple, l'analyse de Rodolphe Morissette, dans Le Devoir, au lendemain des élections du 13 avril 1981. 
rencontre, au Québec, des mouvements d'aspirations sociales et nationales, qui accroîtra l'opposition aux partis traditionnels et toutes les volontés de changement.

En même temps, les différences de nature entre la parti de René Lévesque et le NPD, qui lui tire son origine, entre autres, des organisations syndicales ${ }^{8}$, montrent qu'il n'y a pas une relation automatique entre la recrudescence des luttes sociales et le développement d'une forme particulière d'action politique.

\section{L'alliance non-partisane}

Sous le gouvernement Bourassa, de 1970 à 1976, le syndicalisme semblait engagé dans des voies de rupture avec le régime qui n'étaient pas que conjoncturelles, mais procèdaient d'une trame de fond le conduisant à ne compter que sur ses propres moyens, y compris au niveau de l'action politique. Pourtant, comme nous le verrons maintenant, la progression du $P Q$ va très vite interrompre, ou réorienter, le cours de cette évolution. C'est-à-dire que le rapport entre ce qui paraissait une avancée sur la trajectoire du $3^{e}$ parti, le parti des travailleurs et l'intervention de type non partisan va se résoudre en faveur de cette dernière. Ces logiques contradictoires connaîtront, pendant un court moment, des développements simultanés. Mais rapidement, le pôle retenu sera celui de la non-partisanerie - plus ou moins explicite - pro$P Q$. Non pas un retour à la non-partisanerie d'ancien genre, qui saisissait l'action politique uniquement comme action de pression auprès des gouvernements; mais plutôt une évolution vers des

8. Alors que le Parti québécois, malgré qu'il soit un parti de masse, apparaît d'abord sous la forme d'une scission dans le Parti libéral et d'une sorte d'bémorragie dans l'appareil d'État provincial (Parizeau, Morin, etc...). Graham Fraser a montré (Le parti québécois, op. cit., pp. 62-63) comment, malgré l'adoption de status dont le caractère démocratique tranchait sur ceux du PLQ et de l'UN, les initiateurs du PQ firent en sorte dès le départ d'assurer la prédominance interne des éléments de direction issus de ce processus. 
formules telles que définies par l'expérience nord-américaine alliant l'activité de pression à des alliances électorales stables.

Le fait que la concurrence momentanée des formes d'action politique va se résorber au profit de la non-partisanerie ne peut s'expliquer seulement par l'orientation des directions syndicales: l'ampleur des développements débordent à ce moment le niveau de leur intervention. Nous l'avons souligné, la création du PQ fut saisie comme la réalisation de leur option politique par de larges secteurs ouvriers et populaires. En ce sens, si, analytiquement, les formes de l'action politique des centrales vont être assimilables, à partir de ce moment, aux grandes pratiques non partisanes, la conscience qui émane des processus ne leur est pas réductible. Très clairement, au Québec, c'est le développement d'un parti perçu comme étant indépendant des classes dominantes qui détermine l'orientation et l'action politiques des syndicalistes. D'une certaine manière, les formes réelles de cette action étaient non partisanes, mais la conscience les percevait (pour une bonne part) comme le $3^{e}$ parti réalisé.

La conjonction des revendications de nature nationale et de nature sociale produisait d'ailleurs ses effets propres. Il n'est pas suffisant, pensons-nous, de rappeler que l'extrême tension entre le régime Bourassa et le syndicalisme avait été la cause principale de la chute de celui-là, comme si les luttes sociales seules l'avaient ébranlé. La légitimité et l'autorité du gouvernement libéral furent continuellement minées par une double mise en cause, questionnant à la fois son orientation sur la question nationale ${ }^{9}$ et ses politiques sociales ${ }^{10}$. Ce que le ministre du Travail Cournoyer avait exprimé en 1971 de la façon suivante: "le mouvement syndical devient pour la première fois - un amalgame du mouvement de la classe ouvrière

9. La légitimité même, en fait, des bases de représentation politique sur lesquelles il appuyait son pouvoir.

10. Ouvertement sympathiques à la logique de l'entreprise privée. 
et d'aspirations nationsles» ${ }^{11}$. Il est vrai que les syndicats apparaissent alors comme le véhicule quotidien de l'opposition à Robert Bourassa.

\section{Pouvoir à l'usine}

Durant toute la période qui précède sa première victoire, le Parti québécois se renforce considérablement, tant au niveau municipal que provincial. De plus en plus, les centrales syndicales sont amenées à considérer qu'il occupe tout l'espace de l'opposition politique au gouvernement libéral. Dans ce contexte, alors qu'au cours des années précédentes l'action politique était davantage définie en rapport avec le pourvoir de l'État, (voir les manifestes des centrales CSN, FTQ, CEQ), elle tend dorénavant à être recentrée vers le lieu de travail. Dans le rapport d'Yvon Charbonneau au congrès de juillet 1974 de la CEQ apparaît ainsi la mot d'ordre: Prenons d'abord le pouvoir dans nos écoles ${ }^{12}$. Les thèmes des prochains congrès de la CSN seront: Vivre à notre goût (1974), Prenons notre pouvoir (1976) La CSN aujourd'bui (1977), Élargir nos droits, nos appuis, nos luttes (1978)... Tous ensemble pour de nouveaux pouvoirs (1982). De même, les comités d'action politique deviennent bientôt de simples structures d'appui au $1^{\text {er }}$ front (négociation de conventions ${ }^{13}$ ).

En prévision des élections de 1976, la non-partisanerie emprunta un autre de ses axes traditionnels en Amérique du nord, celui qui consiste à propager (sans parti à soi) ses grandes revendications sous forme de plate-forme législative. La CEQ avait déjà fait allusion à la nécessité de diffuser les revendications des travailleurs p. 219.

11. Cité in Clinton Archibald, Un Québec corporatiste?, Hull, Ed. Asticou, 1983,

12. CEQ, Documents du $24^{\mathrm{c}}$ Congrès, juin-juillet 1974.

13. Cf. l'interview de Jacques Bourdouxe, in L. Favreau et P. L'heureux, op. cit., pp. 101-102; voir aussi les pages 111-122. 
et de les porter sur la scène politique, cependant que Louis Laberge déclarait:

«Je pense que le temps est venu de réunir nos revendications éparses... pour en dégager un véritable programme politique des travailleurs québécois que nous pourrions expliquer à nos membres et... mettre de l'avant au moment des grands débats publics que sont les campagnes électorales" ${ }^{14}$.

À la CSN, le Conseil confédéral de janvier 1976 adoptait une résolution voulant «que les syndiqués, au plan local, régional et national, sur les lieux de travail et dans les fédérations, travaillent à l'élaboration, de l'idéologie politique et d'un programme par et pour les travailleurs " ${ }^{15}$, sans toutefois annoncer de position précise en vue de la prochaine échéance politique. Quand la campagne électorale fut enclenchée, les trois centrales appelèrent plus ou moins ouvertement au vote "pour le parti le plus près de nos intérêts» - ce qui ne pouvait indiquer dans les circonstances d'alors, que le $P Q$. À la FTQ, on invita aussi les syndiqués à militer au sein du Parti québécois, afin d'influencer son programme.

Tous les traits de cette évolution s'affirment aussi dans la période qu'ouvre la victoire du PQ. On met de plus en plus l'accent sur un point de vue voulant qu'il en soit pas de la nature du syndicalisme d'intervenir dans la constitution d'un parti. Norbert Rodrigue, par exemple, tient à préciser au début avril 1976, «que la CSN, comme mouvement syndical, ne doit pas être impliquée dans quelque parti politique que ce soit» ${ }^{16}$, et il complète, au congrès de 1977 par: «l'action politique, c'est (pour nous) l'action revendicative à tous les plans, qui... développe une ligne alternative embrayée sur des changements fondamentaux ${ }^{17}$. De fait, c'est au cœur de ce processus que se confirme pleinement le reflux du pôle « $3^{e}$ parti»: l'action

14. Louis LABERGE, Cible et force de frappe, Montréal, FTQ, 1975, p. 48.

15. Mémo du président Marcel Pépin à tous les syndicats affiliés, 10 février 1976, CSN, Montréal.

16. La Presse, 3 avril 1976.

17. Exécutif de la CSN, Rapport d'orientation: la CSN aujourd'bui, Montréal, 48 congrès régulier, 1977 , p. 38. 
politique se définit sous la forme d'une pression de type socioéconomique sur le gouvernement péquiste, dans une dynamique où la représentation politique du mouvement social est reconnue (ou vue, ou laissée...) au $\mathrm{PQ}^{18}$. Cette orientation est plus poussée à la FTQ qu'à la CSN et à la CEQ. Mais en ce qui concerne les formes de l'action politique, l'attitude est la même.

Il en sera ainsi, encore, à l'occasion du référendum, moment qui concentre l'évolution politique des vingt années antérieures. Sous des formules plus ou moins critiques, le mouvement syndical va se rallier essentiellement à la position définie par le Parti québécois. Attitude identique aussi lors des mobilisations contre le rapatriement de la constitution. Aux élections de 1981, la FTQ appelle explicitement à voter pour le $\mathrm{PQ}^{19}$, alors que la CEQ et la CSN, sans appuyer officiellement ce parti, déclarent que l'objectif prioritaire de la campagne électorale est d'empêcher la victoire du Parti libéral de Claude Ryan.

\section{Institutionnalisation et corporatisme}

Sous le gouvernement péquiste, la non-partisanerie syndicale a aussi recherché le renforcement des mécanismes de contact et de concertation avec les appareils d'État.

Le programme du Parti québécois annonçait une volonté de reconnaissance institutionnalisée du mouvement ouvrier dépassant toute ce qu'on avait connu: promesses d'application universelle

18. Y compris quand la théorisation se fait sous un vocabulaire de gauche et par des secteurs des centrales qui se veulent à gauche des directions: par exemple Michel Doré, du service d'éducation de la CSN (in Pour un syndicalisme combatif, de classe et de masse, Montréal, CSN, mars 1976) se joint quant à lui au rejet du pôle $3^{*}$ parti en soutenant que l'intervention des syndicats dans la formation d'un parti ne peut conduire qu'au réformisme; dès lors, le seul rôle immédiat dévolu au syndicalisme semble aussi un rôle économique, qui devra favoriser cependant les maturations idéologiques (voir p. 18).

19. Première fois, dans l'histoire moderne du Québec, qu'une centrale syndicale appelait explicitement à voter pour un parti non issu du mouvement ouvrier. 
de la formule Rand et de l'atelier syndical, de l'accréditation multipatronale, etc... Dans l'idéologie et la pratique gouvernementale du PQ, cette volonté s'avéra inséparable de la préoccupation d'établir les termes d'une participation structurée des syndicats à la détermination de certaines politiques économiques, de schèmes d'incorporation, en quelque sorte volontaire, de leur dynamique. Ainsi, la période du préjugé favorable aux travailleurs, c'est celle où René Lévesque avance l'idée d'une conférence économique qui, pour la première fois dit-il, réunirait les porte-paroles des principaux agents économiques (discours inaugural à l'ouverture de la session parlementaire, 8 mars 1977); c'est celle de la tenue effective de tels sommets, généraux ou sectoriels, et celle, notamment, où le ministre d'État au développement social, Pierre Marois, soumet l'idée de l'élaboration d'un contrat collectif de base ${ }^{20}$.

On le sait, dans les grands manifestes syndicaux et les proclamations du début des années 1970, le mouvement ouvrier avait aussi reproché au régime Bourassa de ne pas lui reconnaître de rôle véritable dans l'élaboration de ses orientations macro-économiques. La FTQ, par exemple, regrettait qu'avec ce type de gouvernement, attaché aux intérêts financiers, "la consultation n'est pas une forme de pouvoir qu'il concède»; "... le gouvernement portera son nom, ajoutait-elle, le jour où il sera véritablement à l'écoute de la population et des corps organisés qui la représentent"; elle réclamait notamment «la mise sur pied et (l')utilisation rapide du Conseil de planification et de développement du Québec, regroupant des représentants du travail organisé, du patronat et de l'État ${ }^{21}$. Pourtant, le caractère particulièrement conflictuel des rapports syndicats/État avait alors relégué au deuxième plan cet axe d'intervention politique, loin derrière l'articulation des formes d'action contre le gouvernement.

20. Voir Clinton Archibald, op. cit., p. 247.

21. Voir Un seul front, op. cit., pp. 42, 47 et 60; notons que le PQ promit l'élaboration d'un plan à partir d'une concertation d'un type identique à celui avancé ici par la FTQ. 
Rappelons aussi que les centrales québécoises refusèrent unanimement en 1976 le projet de corporatisme tripartite du CTC; même réaction à la fin mars 1977 , quand la direction de la centrale pan-canadienne, suite à une réunion avec des représentants du patronat et du gouvernement à Ottawa, déclara qu'il serait possible d'envisager des restrictions volontaires aux demandes salariales. Pourtant, au Québec, dès l'élection du PQ, des positions favorables à la concertation et aux restrictions volontaires étaient apparues. Des responsables de la CSN, par exemple, avaient pu déclarer:

"Le mouvement syndical pourrait devenir autre chose qu'une machine à revendications si le projet collectif mis de l'avant par le PQ donnait lieu prochainement à des réalisations concrètes...

... je ne vois pas pourquoi les gens de la construction n'accepteraient pas des compromis dans le mode de travail si cela leur permettait en outre de chômer moins longtemps.

Je pense que les syndiqués seraient d'accord pour accepter des fusions de postes (dans le domaine de la santé) qui auraient été impensables sous l'ancien gouvernement...» ${ }^{22}$

À la FTQ, en février 1977, on ouvrit une discussion sur ces questions:

Les militants croient-ils possible que leur centrale s'engage... à réduire certaines revendications salariales? Si oui, à quelles conditions? ${ }^{23}$

Quand le gouvernement Lévesque convoqua son premier sommet, le Bureau national de la CEQ parla d'un «geste politique sans précédent au Québec», pouvant servir «à la consolidation du gouvernement à l'intérieur du Québec, ainsi qu'à sa crédibilité sur les marchés financiers extérieurs ${ }^{24}$. Le mouvement de sympathie était donc présent dans chacune des centrales et il provenait, pensonsnous, de trois facteurs principaux: d'abord, à ce moment, l'ouverture du PQ envers le syndicalisme est un fait réel, au sens où son

22. Le Devoir, 19 novembre 1976.

23. Le monde ouvrier, journal de la FTQ, février 1977.

24. Document du BN pour le Conseil général de la CEQ, 14 mars 1977. 
programme articule les termes d'une institutionnalisation de leur existence qui semblent tout à fait acceptables aux syndicats; ensuite, ses projets de concertation sont présentés non seulement comme faisant une large part au mouvement ouvrier, mais comme visant à redéfinir la vie économique et sociale selon des principes qui apparaissent spécifiquement similaires aux siens; enfin, le parti représente pour l'immense majorité de la base syndicale son parti, celui qu'elle a enfin porté au pouvoir, la promesse du changement; en ce sens, elle est prête à collaborer avec lui, elle lui fait confiance, c'est dans ce cadre précis que l'action politique syndicale tend à accorder plus d'importance aux mécanismes étatiques de participation-concentration.

Le mouvement ouvrier décide de participer aux sommets; retournement crucial de son orientation antérieure, il doit le réaliser formellement, parce que l'invitation du nouveau gouvernement précipite les échéances. Le contexte donnera ainsi lieu à quelques oppositions: si les états-majors des trois centrales se prononcent pour la participation ${ }^{25}$, il y a réticence du côté d'instances intermédiaires ${ }^{26}$. Finalement, sans que les choses soient toutes clairement définies, la présence syndicale est assurée et l'argument majeur invoqué est que les intérêts des travailleurs doivent être défendus à tous les paliers de représentation.

Le plus étonnant dans ces conditions est peut-être que les relations de concertation ne soient pas allées plus loin, ne se soient pas davantage formalisées en des mécanismes stables. Au départ, les bonnes dispositions étaient manifestes tant du côté des syndicats

25. À la CSN, par exemple, il y eut recommandation en ce sens de l'exécutif, du bureau confédéral et du conseil confédéral; cf. le rapport de l'exécutif, La CSN aujourd'bui, op. cit., 1977, p. 3.

26. Les Conseils centraux de la CSN à Montréal et à Joliette, la Fédération des enseignants de Cegep-CEQ, le Bureau fédéral de la FNEQ-CSN et quelques syndicats locaux. 
qu'au sein du gouvernement. Mais plusieurs facteurs allaient jouer contre la concertation. Premièrement, la situation économique marquée par la récession; si le gouvernement promulgue des réformes allant dans le sens des positions syndicales ${ }^{27}$, il ne s'engage pas sans réserve dans le type de concessions ${ }^{28}$ qui, à terme, pourraient véritablement approfondir la concertation avec les centrales. Ensuite, le bastion le plus puissant du mouvement ouvrier au Québec, celui qui, dans la dernière période, a servi de volet d'entraînement et donné le ton à l'évolution syndicale est constitué de l'organisation dans la fonction publique et para-publique. Or dans les vingt dernières années, ce secteur déterminant a été en rapport de négociation avec l'État-patron, ce qui a contribué à accentuer sans cesse les points de friction syndicats-gouvernement. La décision de la CEQ de ne pas participer en mars 1979 à la deuxième conférence socio-économique au sommet (Montebello), alors que s'ouvre la nouvelle ronde de négociations du Front commun, trouve ici ses tenants. Surtout que les difficultés économiques semblent avoir amené le gouvernement à modifier sa position initiale: alors qu'il demandait, en quelque sorte, aux dirigeants des centrales de jouer un rôle de médiation auprès de leurs groupes affiliés, il leur demande (en 1978-1979) d'assurer une forme de modération des revendications ${ }^{29}$. Enfin, les mécanismes participationnistes sont entrés en crise aussi du simple fait que le mouvement ouvrier s'était illusionné sur la nature du programme péquiste. On attendait du gouvernement des mesures économiques

27. Encore ces réformes soient inégalement appréciées selon les centrales, la FTQ se montrant en règle générale plus satisfaite: i.e., loi sur les accidents de travail, loi anti-scab, etc...

28. L'exemple de la loi 290 dans la construction est vu comme une référence à cet égard par le mouvement syndical.

29. Si nous nous inspirons des catégories analytiques articulées par Gregor Murray, Trade-Union strategies vis-à-vis the State, communication présentée au congrès de l'Association canadienne de science politique, Guelph, été 1984. 
et sociales ${ }^{30}$ et une démocratisation de l'État ${ }^{31}$ qui exigeraient des niveaux de rupture avec l'ancien régime, aussi bien qu'avec l'État fédéral, qu'il n'était pas prêt d'envisager.

Les processus «d'incorporation» du mouvement ouvrier resteront à l'état de projet, malgré des tentatives évidentes en cette direction de la part du gouvernement. Les rencontres au sommet seront même interrompues un an avant le référendum, peut-être parce qu'elles en venaient à montrer davantage l'écart entre les positions des «partenaires» que les points de consensus.

Mais l'impact de ces développements sur les attitudes politiques du mouvement ouvrier organisé, et sur celles de ses membres, fut moins important qu'il n'y pourrait paraître à première vue. Le statut du Parti québécois ne relevait pas d'abord d'une exacte correspondance entre son programme social et celui des syndicats. Il faut à nouveau le souligner: la place du PQ procédait premièrement du contenu dont étaient chargées les aspirations nationales, et du monopole de représentation politique qu'il avait réussi à exercer sur ces aspirations. À l'époque, c'était la marche au référendum qui ponctuait fondamentalement la conscience politique.

30. Par exemple, à son congrès de 1977 où elle définit déjà la situation économique comme étant la pire depuis la crise des années 1930, la CSN demande la création de secteurs économiques d'entraînement - en utilisant au besoin l'arme de la nationalisation, l'établissement d'un système bancaire québécois, la transparence-économique des entreprises, etc... voir L. Favreau et P. L'Heureux, op. cit., pl 128.

31. Dans leur Mémoire commun sur le projet de loi 53, présenté au gouvernement en décembre 1977, les trois centrales soulèvent par exemple, l'idée d'une transparence de la fonction publique, que les dossiers et documents préparés par les employés de l'État, notamment ceux du ministère du Travail et de la Commission des accidents du travail, devraient circuler librement et alimenter le débat public, qu'on devrait séparer la fonction publique des préoccupations des partis au pouvoir et la lier davantage à la population... Comme il ne le fait pas, on dit que le PQ ne respecte pas ses promesses électorales... (voir p. 2 sqq.). Pour une analyse plus globale des tentatives de concertation du Parti québécois, se référer au chapitre 8 du livre de Clintion Archibald, Un Québec corporatiste?, op. cit... 
Dans ce cadre, les mécanismes de contact entre l'État provincial et le mouvement ouvrier ne disparurent pas: ils changèrent plutôt de registre. Il est révélateur que l'accès au cabinet péquiste d'hommes et de femmes liés au syndicalisme semble s'accentuer au moment où les sommets économiques s'avèrent moins prometteurs. Un peu comme si on avait voulu établir des voies privilégiées et supérieures de liaison qui échappent à la tourmente publique, favorisent le règlement de différends et structurent la concertation au plus haut niveau ${ }^{32}$. Le fondement prioritaire de l'action politique du mouvement ouvrier restait alors centré sur sa volonté de participation à l'élaboration des grandes politiques de l'État. De 1976 à 1982, le PQ a réussi à préserver son hégémonie générale sur l'activité ouvrière et populaire notamment en période électorale.

\section{La rupture de l'alliance non-partisane}

La victoire du Parti québécois en 1976 n'était pas assimilable, avons-nous dit, à un simple processus traiditionnel d'alternance gouvernementale. Elle apparut comme une victoire du vote populaire, la première ou, assurément, la plus importante qu'il ait jamais remportée sur le terrain électoral.

Ce sont ces données qui permettent de saisir toutes les répercussions de la défaite référendaire, puis le coup terrible que constitua le rapatriement unilatéral de la constitution. Ces deux événements marquèrent l'échec du péquisme sur la question (nationale) qui était devenue le centre de gravité des volontés de changement et, de ce fait, avait expliqué premièrement et ultimement le ralliement au Parti québécois. En ce sens, l'enclen-

32. Si Robert Burns (de la CSN) est membre du $1^{\text {cr }}$ cabinet Lévesque, c'est après 1979 que les Clément Richard (CSN), Guy Chevrette (CEQ), Robert Dean (FTQ) entrent au Cabinet. Au fil des ans, la FTQ s'est avéré la centrale la plus liée au gouvernement Lévesque; elle est probablement celle pour laquelle l'hypothèse ici esquissée s'applique le plus directement. 
chement d'un processus de rupture avec le PQ devait d'abord inclure, dans son déroulement, un constat d'échec sur sa politique concernant la question nationale. C'est ce qui commença à se dégager du moment politique 1980-1982.

Gérald Larose, par exemple, qui dirigeait alors le Conseil central de la CSN à Montréal, et qui est maintenant président de la centrale, déclara peu après le référendum que le 20 mai 1980 avait été la victoire de «la formidable coalition des forces de la réaction capitaliste, patronale et fédéraliste», et il remit ouvertement en question le monopole qu'avaient consenti les syndicats au PQ sur la question nationale ${ }^{33}$. Yvon Charbonneau (CEQ) conclut son analyse de la défaite référendaire de la manière suivante: "Aussi, il importe plus que jamais que mûrissent et surgissent certaines propositions actuellement en gestation d'action politique autonome des travailleurs et des classes populaires» ${ }^{34}$. Même conclusion, et presque dans les mêmes termes, du côté d'André Leclerc, permanent à la FTQ et l'un des conseillers de la direction sur l'action politique ${ }^{35}$.

De fait, dès après le référendum et pour la première fois depuis 1970-72, resurgissent les questionnements sur les formes d'action politique du mouvement ouvrier. Mais ce sont des questionnements qui, à ce stade, tout en étant significatifs, apparaissent comme signes avant-coureurs d'un développement embryonnaire. La vie politique est alors tout absorbée par l'opposition au rapatriement, enjeu qui reproduit puissamment la pression du lien avec le Parti québécois. En 1981, la FTQ appellera à voter pour le PQ cependant que la CSN et la CEQ demanderont, on l'a dit, d'éviter le pire - c'est-à-dire d'empêcher l'élection du PLQ de Ryan, seul opposant à René Lévesque. Mais avec le rapatriement, l'impression d'un échec de toute l'orientation constitutionnelle

33. Gérald Laorse, "Les syndicats et le référendum», in L'impasse, p. 61 sqq.

34. Yvon Charbonneau, "D'octobre 1970 à octobre 1980 », in L'impasse, id., p. 60.

35. André Leclerc, "Les lendemains du lendemain qui n'a pas chanté», in L'impasse, id. voir. p. 44. 
suivie par le gouvernement péquiste grandit. Les critiques s'élèvent à un degré qu'on n'avait pas vu lors du référendum. Des signes réels, limités toutefois dans leurs conséquences immédiates, de distanciation politique entre les centrales et le Parti québécois sont exprimés aux plus hauts échelons. Le président de la CEQ, Robert Gaulin, explique que la stratégie politique du PQ a servi de «marche-pied» au coup de force du premier ministre Trudeau, alors que la FTQ, la CSN et la CEQ signent une déclaration commune $^{36}$ dont le contenu remet en cause, au moins implicitement, l'orientation du gouvernement Lévesque. Elles y déclarent, entre autres choses:

"Comme le processus actuel joue toujours contre nous, nous devons nous donner un mode de détermination constitutionnelle qui nous convienne; le seul... c'est celui qui consiste à redonner la constitution à celui à qui elle appartient, c'est-àdire au peuple lui-même... (La) seule façon pour le Québec de sortir du gouffre actuel, c'est de s'appuyer sur le peuple organisé, rassemblé...»

Que le peuple québécois élabore et adopte sa propre constitution, cela contredit en effet une stratégie qui a misé sur l'accord constitutionnel avec les premiers ministres des autres provinces, les appels à la Chambre Haute de Grande-Bretagne, etc... Et l'importance d'une telle déclaration vient de ce que jusque là on avait, pour l'essentiel, laissé au parti de René Lévesque la détermination des termes et des stratégies de l'intervention sur ces questions.

Mais à ce moment, le Parti québécois pouvait encore apparaître comme différent des Libéraux. La crise économique cependant, précipita les événements. Après son échec sur la question nationale, le PQ adopta des politiques unanimement considérées par le mouvement ouvrier comme reaganiennes. L'invocation du nationalisme ne suffisait plus à contenir l'opposition des syndicats. Il y eut

36. En collaboration avec l'Association québécoise des professeurs de français et la Société Saint-Jean Baptiste de Montréal, en décembre 1982, suite au jugement de la Cour suprême établissant la légalité de la démarche du gouvernement fédéral. La déclaration de Robert Gaulin date d'octobre 1980. 
affrontement direct. L'ampleur de la désillusion politique postréférendaire, à notre avis, accrût la colère et la détermination des syndiqués.

Nous ne pouvons, dans le cadre de cette étude, revenir sur l'ensemble des péripéties qui marquèrent les relations PQ-syndicats en 1982-83 37 . Rappelons qu'elles furent d'abord marquées par la volonté du gouvernement Lévesque de revenir sur des acquis syndicaux dans les secteurs publics et para-public, de réouvrir à la baisse des conventions collectives en vigueur, puis de reprendre une part des salaires que ses employés avaient déjà touché: les lois 70, 105 et 111 consignèrent ces mesures, ainsi que l'interdiction du droit de grève, le décret des conditions de travail (avec augmentation de la tâche, par exemple, chez les enseignants), une suspension des libertés civiles sur ces questions, etc...

Il faut prendre la mesure de cette évolution: c'est contre le gouvernement péquiste, qui avait soulevé tant d'appuis, que le mouvement syndical appelait au déclenchement d'une grève générale et illégale, le 9 janvier 1983. Il y avait rupture. L'articulation immédiate de cette rupture trouva son déroulement majeur avec la grève des enseignant-e-s et des employé-e-s professionnel-le-s de l'État au printemps 1983, quand le gouvernement menaça ouvertement les syndicats impliqués de les désaccréditer, d'abolir le précompte syndical, et d'imposer à chaque gréviste la perte d'une année d'ancienneté pour toute journée d'arrêt de travail illégal, ou encore de congédier en bloc les professionnel-le-s du gouvernement (menace inspirée de la mesure prise par le président Reagan contre les contrôleurs aériens, aux États-Unis).

Les voies mêmes de l'institutionnalisation du mouvement syndical étaient à nouveau mises en cause. Le Parti québécois ne pouvait plus apparaître comme le véhicule politique du mouvement

37. Serge Denis a essayé d'en suivre les principaux cheminements in «Développement, tensions et lignes de clivage du mouvement ouvrier au Canada", Crise économique, transformations politiques et changements idéologiques, Cahier de l'ACFAS, no. 16, 1983, pp. 373-398. 
social. Dans les mois qui suivirent, le poids des aspirations nationales et des aspirations sociales se conjugua à nouveau, mais son efficacité jouait cette fois contre la crédibilité du parti de René Lévesque. Cela produisit des tensions au sein même du PQ, puis des démissions chez ses députés et ministres (davantage, mais pas uniquement, sur les revendications d'ordre national), l'imposition de mesures draconiennes de discipline à l'intérieur du parti (qui ont durablement ébranlé son auréole de démocratie interne), et surtout un véritable effondrement du nombre de ses membres: le PQ passa de quelque 300,000 membres en cours d'année 1981 à moins de 80,000 au premier trimestre de 1985 , dont probablement pas plus de 50,000 véritablement en règle.

D'une certaine manière, le niveau des attentes non satisfaites et même niées mesure l'ampleur de la débâcle.

Ces différents facteurs secouent bien évidemment toutes les modalités de la non-partisanerie sur lesquelles le mouvement syndical a établi son rapport à la politique. Mais ils ne jouent pas seuls.

\section{Après le 2 décembre $1985 \ldots$}

La spécificité de la situation économique pèse de tout son poids sur le mouvement ouvrier. L'ampleur des problèmes exige des interventions qui débordent le cadre des contrats de travail. La nécessité d'un accès renfrorcé aux mécanismes et aux processus d'élaboration des grandes politiques étatiques devient plus pressante ${ }^{38}$. Mais comment, par quelle voie l'assurer? Cet enjeu devient central au moment où le canal privilégié de représentation

38. Par exemple, le document Pour le droit au travail, préparé par les Services de l'action politique et de la recherche de la CSN, et soumis au Conseil confédéral de la centrale des 8-12 décembre 1981, décrit toutes les politiques actuelles des gouvernements comme inefficaces à contrer les conséquences les plus répandues de la crise, sans parler de leur incapacité à contrer cette crise elle-même (pp. 16-18; 53-77). Il situe par ailleurs directement au niveau politique les possibilités éventuelles de solution, et met en avant un ensemble de revendications qui nécessitent l'intervention immédiate de l'État (lois, modifications des rapports de l'État aux entreprises, etc...). 
politique qu'avait paru être le PQ s'est brisé et où les lieux de participation-concertation syndicats-État sont affaiblis. La forme unique (non-partisane) d'action politique développée par le syndicalisme québécois est soumise dès lors à de fortes tensions, surtout quaucune organisation représentative ne pense trouver du côté du gouvernement libéral une véritable voie alternative.

Nous en arrivons à un point névralgique de l'évolution de la situation politique depuis vingt ans.

Dans la mesure où le processus de rupture avec le Parti québécois continue de se développer - et il faudra voir s'il se confirme au cours des prochains mois - c'est à nouveau la question de la restructuration d'une alternative qui est appelée à ressurgir.

Les libéraux eux-mêmes, contrairement aux années ' 60 et même ' 70 , ne se présentent nullement sur un programme de concessions aux forces syndicales et populaires.

Dans ces conditions, comment et sur quelles bases, pourra se maintenir au cours des prochaines années la ligne de nonpartisanerie du mouvement syndical?

Parmi les formations politiques créées à l'extérieur du mouvement ouvrier, depuis les années 50, le Parti québécois est apparu comme la force la plus progressiste. Or, c'est cette image que les dernières années ont effacée. Le Parti libéral ne peut, ni visiblement, ne veut, la revendiquer pour lui. Dès lors, à quel parti existant le mouvement syndical peut-il encore tenter d'articuler de manière stable sa ligne politique des vingt dernières années?

La question refait surface avec d'autant plus d'acuité que l'horizon de la scène politique paraît bouché. Et de la difficulté d'y répondre, mais aussi de la nécessité d'y répondre à terme, jaillissent les tensions et les interrogations qu'on a vu se manifester tout récemment dans les syndicats.

Les prises de position dans les centrales se sont accentuées depuis quelques mois pour un renouvellement de l'action politique. En témoignent, notamment, les documents et interventions de 
la Fédération nationale des Communications (CSN) ainsi que les débats au dernier congrès de cette centrale, de même que la décision du congrès de la FTQ, en novembre 1985, de rejeter la recommandation d'appui au PQ formulée par la direction ${ }^{39}$.

Ces prises de position posent en elles-mêmes le problème de l'absence de représentation organisée du mouvement syndical sur le plan politique. Elles font, soit explicitement, soit implicitement, une critique de la non-partisanerie comme forme d'existence politique insuffisante et inadéquate du syndicalisme dans la situation économique et politique présente. Elles indiquent la reprise des discussions sur l'engagement politique du syndicalisme, notamment en période électorale.

Il est impossible de prédire l'évolution prochaine de ces développements, évolution qui de toute façon ne peut pas s'entrevoir comme un processus linéaire.

Une chose nous paraît évidente néanmoins. Pour deux raisons principales, la situation actuelle ne met pas à l'ordre du jour un simple retour aux questions politiques de la fin des années 1950 et du début des années 1960 .

D'abord, le mouvement syndical est considérablement plus fort en termes de membres et d'organisation, même si sa capacité de riposte face à la crise économique a été durement éprouvée. La force concentrée qu'il représente poserait à elle seule la question de son rôle comme levier pour la constitution d'une nouvelle organisation politique en des termes nouveaux par rapport à ceux d'il y a vingt-cinq ans.

D'autre part, si au cours des vingt dernières années le syndicalisme a pu, sur la base des concessions qu'il obtient, ordonner son action politique autour d'options structurées à l'extérieur de

39. Fédération nationale des communications, "Les enjeux du prochain congrès de la CSN, mars 1985; Conseil central de Montréal (CSN), "Pour une action politique syndicale», mars 1985; Fédération des travailleurs et travailleuses du Québec (FTQ), congrès de novembre 1985 . 
son propre mouvement, les conditions à cet égard ont eu tendance à s'épuiser depuis le début des années 1980.

Jamais n'avait-on mis en place une solution politique soulevant autant d'attentes que celle du PQ. Mais par le fait même, la question politique a tendance à ressurgir présentement dans les syndicats non pas comme il y a vingt ans, avant que l'expérience de cette solution ait été faite, mais sur la base même de cette expérience. Cela, à terme, est un facteur supplémentaire de maturation, et annonce l'ouverture d'un débat de fond sur l'ensemble des aspects reliés à l'organisation et à la représentation politiques du mouvement syndical québécois. Nettement, aujourd'hui, une page de l'histoire politique du syndicalisme au Québec semble avoir été tournée. 